\title{
Regularities of the day surface deformation during layer mining by consecutive lavas
}

\author{
Tamara Makeeva ${ }^{1, *}$, Vitaly Trofimov ${ }^{2}$ \\ ${ }^{1}$ Moscow State University of Civil Engineering, Yaroslavskoe shosse, 26, Moscow, 129337, Russia \\ ${ }^{2}$ Russian Academy of Sciences, Institute of problems of complex development of bowels, 111020, 4, \\ Kryukovsky tupik, Moscow, Russia
}

\begin{abstract}
The main objective of this work is to find an analytical ratio, which could describe the form of a trough to simplify the forecast of deformation of a day surface during the development of underground mining operations. The interactive algorithm for finding of the defining parameters of such a ratio, which allows to expand the borders of the use of traditional approach significantly, is offered. The example of interpretation of the data, obtained within the measurement of subsidence of a day surface in the mine named after Kirov in Kuzbass during consecutive mining of two lavas is reviewed. The analytical ratio for the description of a form of a trough from one lava is received and proved to be fair for the following lava; that gives the basis to consider it fair for the subsequent mining of the layer.
\end{abstract}

\section{Introduction}

The forecast of deformation of a day surface during underground mining operations is a very relevant task.

Great contribution to studying of processes of deformation of the day surface during mining and to the creation of a methodological basis for the of assessment was brought by S.G. Avershin, D.A. Kazakovsky, R.A. Muller, M.A. Iofis, V.N. Gusev, V.F. Podakova, Yu.A. Limanova, S.N. Silvestrova, M.V. Dolgikh, E.M. Volokhova, V.P. Hutsky, S.V. Mazein, M.A. Karasyov, V.V. Rechitsky, D.V. Panfilov, etc.

However the existing methods of the assessment of displacement and deformations do not exhaust the subject. The algorithm, which would allow to investigate the features of formation of a trough of subsidence of the day surface, caused by underground mining, is quite necessary.

\section{Methods and algorithms}

Within the known restrictions it is supposed, that the vertical subsidence of the land surface is described by the following three-parametrical function rather well [1-5]:

\footnotetext{
Corresponding autour: makeeva13new@yandex.ru
} 


$$
\eta(x)=-\frac{\eta_{0}}{2}\left[F\left(\frac{D_{p}+x}{C_{p} H}\right)+F\left(\frac{D_{p}-x}{C_{p} H}\right)\right],
$$

where $\eta_{0}, D p, C p-$ the constants characteristic of specific conditions, $\Phi(t)=\sqrt{\frac{2}{\pi}} \int_{0}^{t} e^{-\frac{z^{2}}{2}} d z^{-}$ integral of probabilities of Gauss, $H$ - depth of the horizontal development, $x$ - the coordinate along the land surface, counted from the middle of a trough.

At the same time: $\eta_{0}$ - the volume of the maximum subsidence of a roof of development in case of full undermining, which is usually connected with the layer power $m$; $D_{p}$ - the parameter, which correlates with the value of opening length and makes up $\sim 0.7 \div 0.9$ of it; $C_{p}$ - the parameter, connected with the mechanical properties of the leaning breeds and the possible mechanism of their deformation.

The form of the day surface subsidence in each particular mining situation is defined by the parameters, which have to be determined according the values of displacement of reference points, with the use of a suitable algorithm.

Thus, the task consists in the choosing of a function of a definite type (1), or in other words in choosing the volumes of $D_{p}, C_{p}$ so that all $\eta_{i}$ were close to the corresponding curve (1).

$$
F=\frac{1}{N} \sum_{1}^{N}\left[\eta_{i}-\eta\left(x_{i}\right)\right]^{2}
$$

to which so-called method of least squares is bound. Parameters $D_{p}, C_{p}, \eta_{0}$ have to be chosen so, that the functional $F$ would be as minimal as possible. In theory this minimum is equal to zero.

Let us write out the type of the functional of $F$, taking into account features of the considered task. From (1) and (2) we can suppose:

$$
F=\frac{1}{N} \sum_{1}^{N}\left\{\eta_{i}+\frac{\eta_{0}}{2}\left[F\left(\frac{D_{p}+x}{C_{p} H}\right)+F\left(\frac{D_{p}-x}{C_{p} H}\right)\right]\right\}^{2},
$$

Thus the task comes down to the search of the minimum of function (3) for variables $D_{p}, C_{p}, \eta_{0}$.

Such searching can be carried out both within numerical and analytical approach. During numerical searching of a minimum we will mention the well-known and worked in details multistage algorithms of coordinate-by-coordinate and gradient descent, which always leads to the required decision.

At the same time very efficient and rather simple approach to searching of the minimum of the functional, which is based on ideology of methods of "random search", can be used. In its framework there is a set of methods, significantly multistage, which main feature is the random sampling of a point of approbation of the given function [6].

The mentioned algorithms in essence solve the objective of the best approximation of the experimental data on subsidence of the land surface, with the curves of the given type (1). At the same time a question still remains, how good is this best approximation. The offered algorithms comprise the parameter, which can serve as the criterion of accuracy of approximation; this parameter is the square norm (4). The numerical value of the functional (4) reflects the measure of deviation in the valley in total of the experimental and estimated 
values of shifts. At the same time, the square root from $F$ represents the average standard deviation of the experimental points from the found curve (1).

\section{Results}

The research $[3,4,6,7]$ completed by the authors proved that the function (1) approximates the measured value of subsidence of the reference marks located over underground framings, well. The average square deviation for all the reference marks of the measuring station can make up $\sim 1 \div 2 \mathrm{~cm}$ (and less).

However in many cases considering the curves of subsidence proves the existence of some fracture of these curves at some distance from the trough centre. The attempts of creation of a uniform curve (1) by the means of the algorithms, mentioned above, lead to a systematic discrepancy of this curve and the experimental points.

In this regard the interactive algorithm of the creation of functions (1), that allows to change the parameters $D_{p}, C_{p}, \eta_{0}$ directly on the display by the means of a computer mouse and visually to control the compliance to the received curve to the selected set of the experimental points, was developed. The screenshot of this program is presented in Figure 1. The current parameters $D_{p}, C_{p}, \eta_{0}$ value, the appropriate square root deviation and its minimum value in the course of calculation are displayed. According to the results of the program runtime the estimated form of a trough of subsidence is output into a separate file.

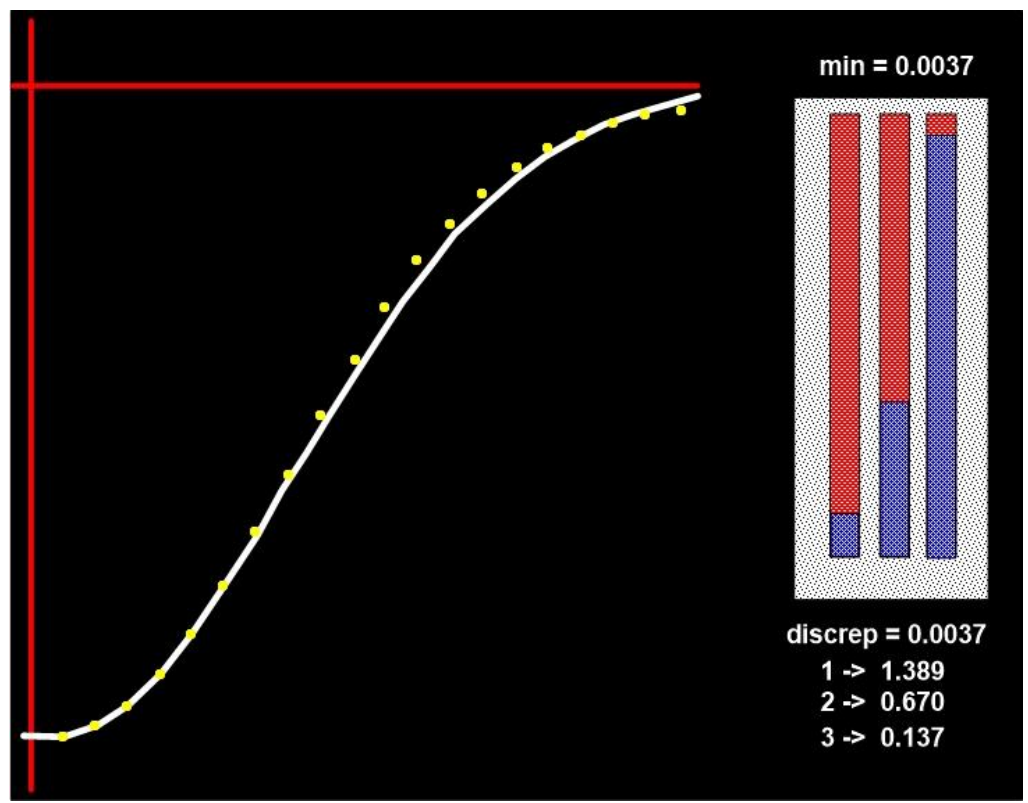

Fig. 1. Screenshot of the interactive program

Such algorithm allows to construct the curve (1), which is partially approximating the experimental data only in the top or in the bottom of a trough. That is demonstrated in the figure 2 . 

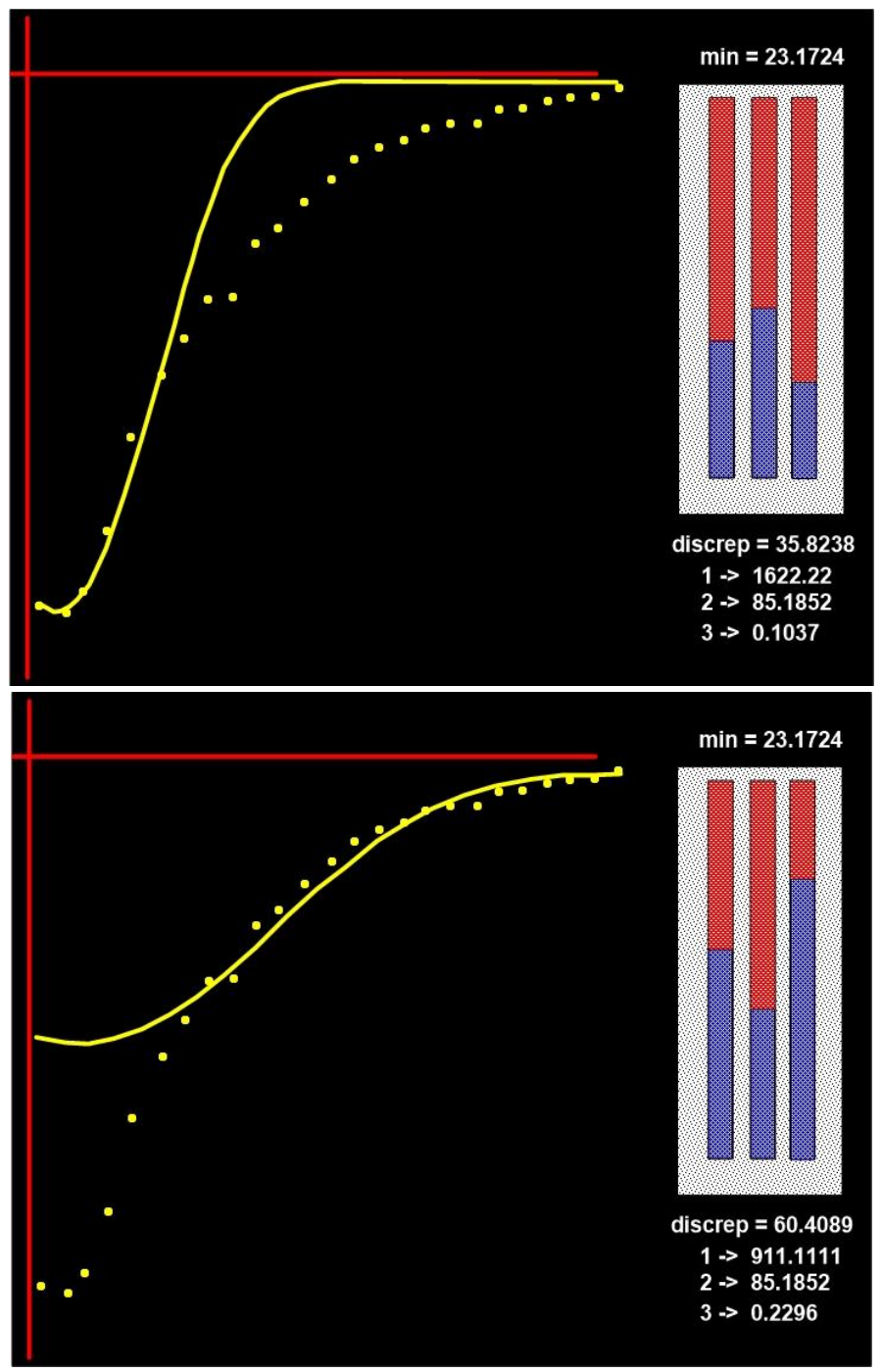

Fig. 2. Selective approximation with the use of the interactive program

Thus, the offered algorithm has practical value.

\section{Discussions}

Let us consider the following case from practice. During mining of two lavas on the Boldyrevsky layer (the mine named after Kirov, Kuzbass) the subsidence of the day surface was controlled by the fiducial station, consisting of 60 reference marks. Schematically the mining situation is presented in the figure 3 . 


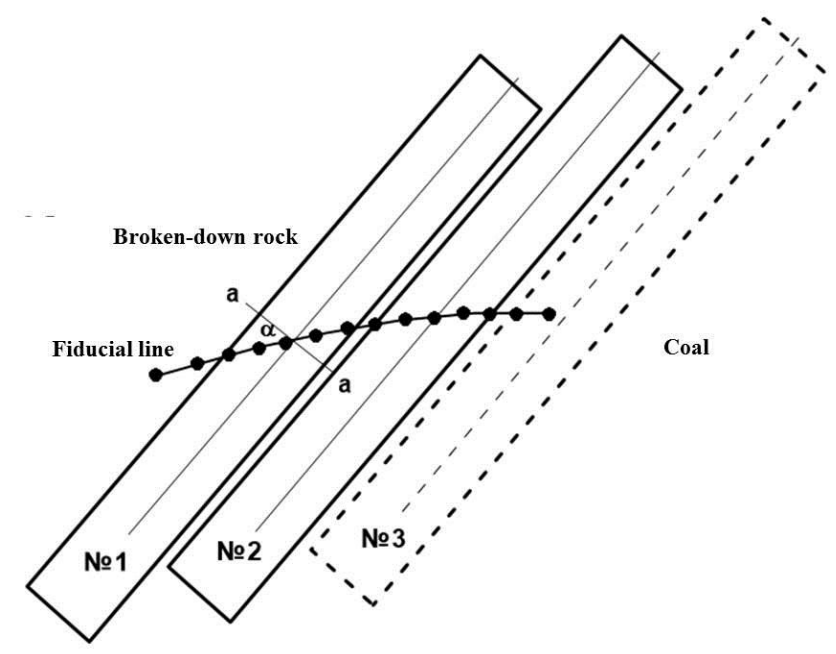

Fig. 3. Scheme of serial mining of three lavas

We shall mention, that in considered case the line of reference marks is not perpendicular to the direction of lava advance, and that is necessary for applicability of ratio (1) [3-7]. In this regard the adjustment of values of coordinates of the reference marks layout and their coercion to a straight line $a-a$, which is perpendicular to the axis, is necessary. It is obtained by multiplication of values of $x_{i}$, which are digitized along the fiducial line, and $\cos \alpha$, i.e. $\cos 57^{\circ}=0.54$. Such corrected curve is presented in Figure 4.

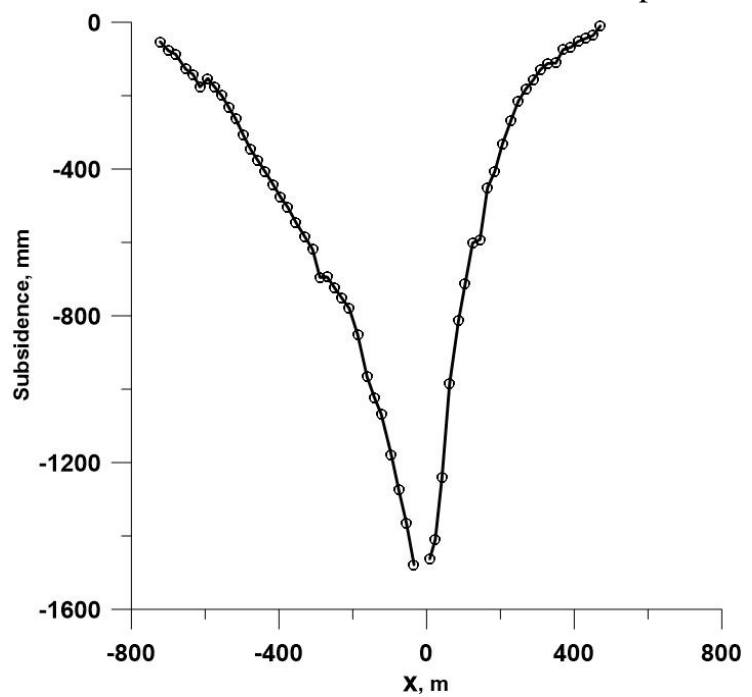

Fig. 4. Form of the trough subsidence for lava No. 1 in its middle part

We shall mark, that it is asymmetrical concerning the axis of the taken-out pole. It is caused by the fact that the properties of breeds on the left and on the right of the pole are various. There is an array of the brought-down breeds on the left as the result there coal has been taken out earlier, and there is still untouched coal on the right, which will be taken out 
by the subsequent lava No. 2. For this reason the approximating functions shall differ on the right and on the left.

Figure 5 demonstrates the attempt to approximate the curves in Figure 3 with the uniform functions (1) on the right and on the left, where the experimental data are marked by characters (o). Though these curves deliver minimum to the functional $F$, but their discrepancy from the experimental points both in the upper part, and in the lowest part of a trough is visible.

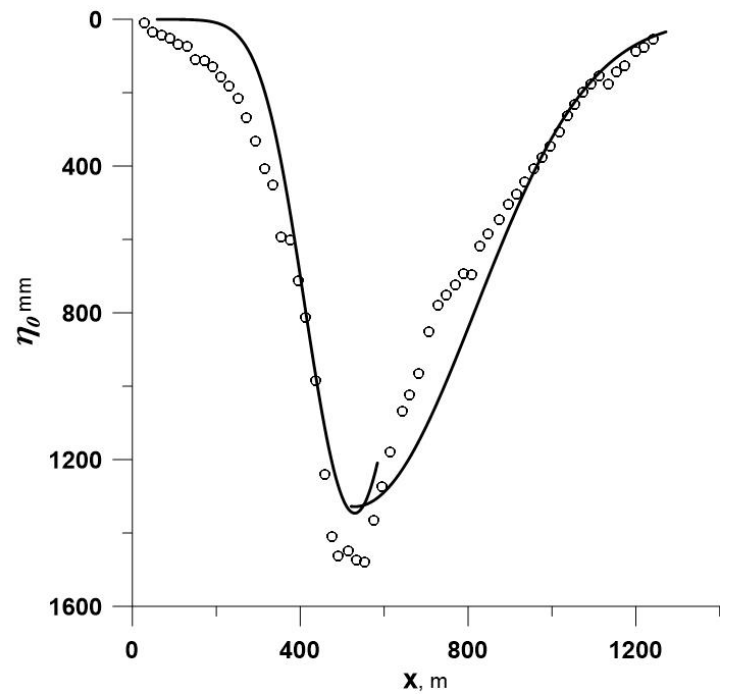

Fig. 5. Approximation of the reference points shifts with the uniform curves on the right and on the left

Fractures on the experimental curves, both on the right curve, and on the left curve, share the trough on upper part (with more slanting bank) and lower part (with more steep bank). By the means of the interactive algorithm 4 curves, which bring closer the experimental data on the appropriate sections in the best way, were constructed. They are presented in Figure 6a.

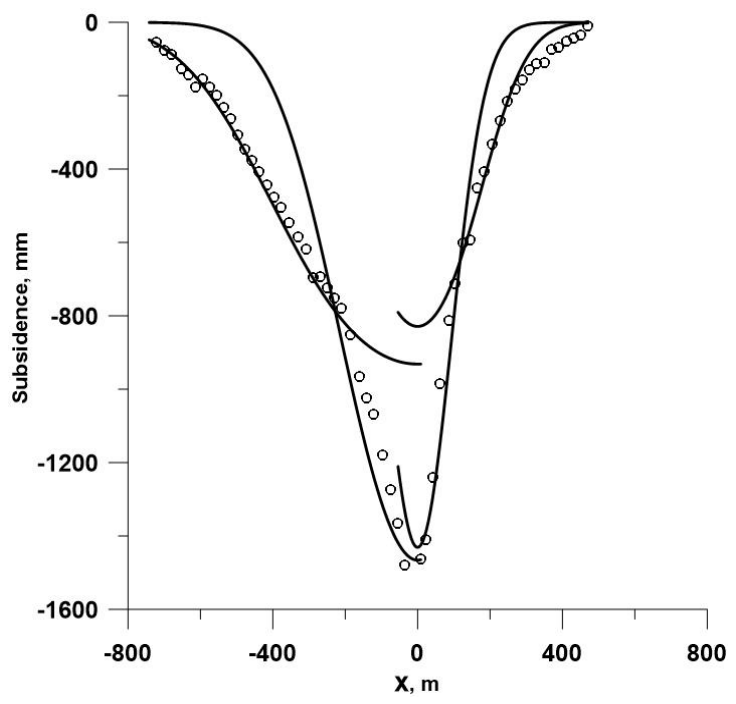

a) 


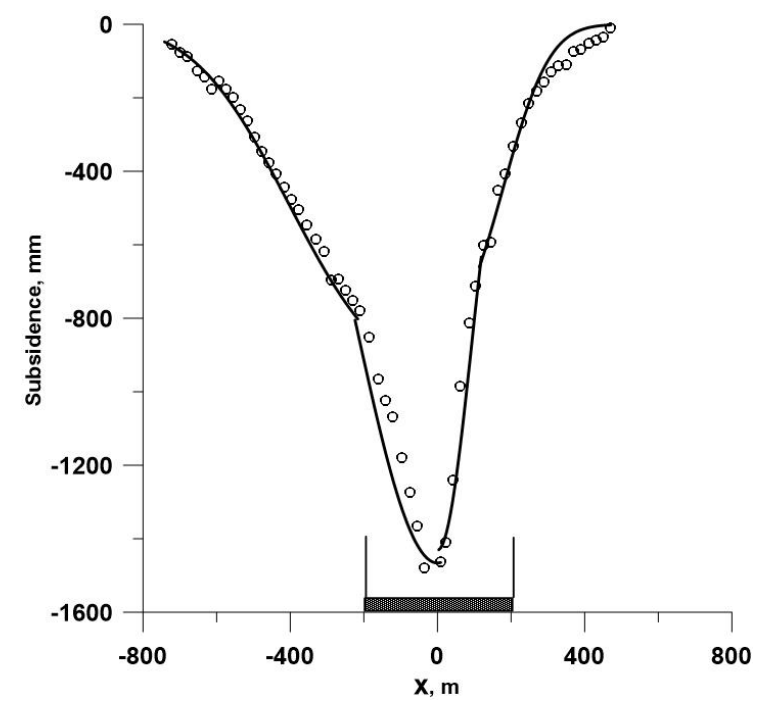

b)

Fig. 6. Approximation of data of the reference points shift on separate pieces

Figure $6 \mathrm{~b}$ presents only significant parts of these curves in comparison to the experimental points. General analytical expression can be written out for them, though the parameters, entering it, are various for different parts of a trough. They are presented in Table 1.

$$
\eta(x)=-\frac{\eta_{0}}{2}\left[F\left(\frac{D_{p}+x}{C_{p} H}\right)+F\left(\frac{D_{p}-x}{C_{p} H}\right)\right]
$$

where:

Table 1. Parameters for different parts of a trough

\begin{tabular}{|c|c|c|c|}
\hline & $\eta_{0}$ & $D_{p}$ & $C_{p}$ \\
\hline$x<-200$ metres & 911 & 170 & 0.23 \\
\hline$-200<x<0$ & 1622 & 85 & 0.1 \\
\hline $0<x<200$ & 2956 & 52 & 0.267 \\
\hline$x>200$ metres & 1067 & 144 & 0.156 \\
\hline
\end{tabular}

We shall mention the following circumstance. Figure 6 demonstrates the boundaries of the developed space (lava width). It is visible, that the fractures of the curves of the subsidence are located approximately over these boundaries. In this regard it is possible to assume, that the deformation properties of the breeds over the developed space and out of it differ noticeably.

It is known $[5,8,9]$, that the considerable zone of the array unloading, which is spread up to the day surface is created over the developed space. Unloading leads to the breeds loosening, and perhaps to disclosure of cracks to some extent, and as a result to reduction of their deformation and strengthening properties. 
The received results, such as ratio (5) with the appropriate table, can be used for the forecast of a day surface subsidence in case of mining of the subsequent lavas, because the mining situation for them completely repeats the same situation for already considered lava.

After mining lava No. 1, the regularities of formation of the trough for which have been considered earlier, one more lava (No. 2) was mined. For it Samplings of the subsidence of a day surface were carried out for it with the use of the same fiducial station (curve 2, figure 7).

In the figure 7 the day surface subsidence for both lavas are combined for comparison curve. It is visible, that they completely coincide from untouched coal whereas there is some divergence from the brought-down breed.

Such coincidence gives the chance of rather confident prediction on subsidence during mining of the following lava No. 3 .

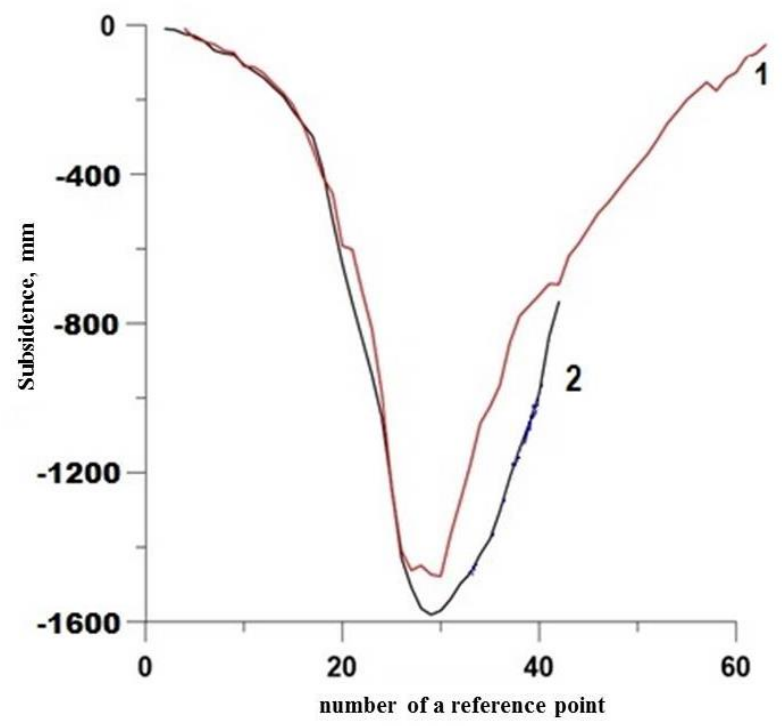

Fig.7. Combination of the curves of the day surface subsidence for both lavas

\section{Conclusions}

The algorithm, presented in this work allows to investigate the features of formation of a trough of the day surface subsidence, caused by the underground mining in details. At the same time the standard function stated as (1) is used, but the parameters, defining it, are not constant and have patch distribution among the trough sections in the form of four pieces. Such inhomogeneity, probably, reflects the distinction of properties of breeds over the mining space and out of it, which is caused by technogenic influence. Breeds over mining are partially unloaded from mountain pressure, and that leads to disclosure of cracks and stratification, and, as a result, to the loss of resilient and strength properties.

Besides, it is necessary to consider the fact, that mining lava usually happens in addition to the already waste site of coal layer. In this regard the section of a trough has the asymmetrical form, concerning its axis. The given algorithm is also intended for solving problems with such asymmetry.

The main problem, which was set during work, is to try to find some analytical relations, which could describe the form of a trough for the actual mining situation in order 
to simplify the forecast of the day surface deformation during further underground mining operations.

\section{References}

1. G. Cratch, Movement of rocks and protection of underworked structures (Moscow, Nedra, 1978 )

2. R. Muller, Influence of excavations on deformation of the land surface (Moscow, Ugletekhizdat, 1958)

3. S. Kuznetsov, V. Trofimov, Marksheyderiya of Kazakhstan: state and prospects, 1, 119125 (2006)

4. S. Kuznetsov, V. Trofimov, Gorny journal of Kazakhstan, 7 ( 2006)

5. S. Kuznetsov, V. Trofimov, FTPRPI Journal, 4, 1-22 (2007)

6. T. Makeeva, V. Trofimov, MATEC Web of Conferences, 117, 00107 (2017).

7. T. Makeeva, V. Trofimov, IOP Conf. Series: Materials Science and Engineering, 365, 042025 (2018) doi:10.1088/1757-899X/365/4/042025.

8. S. Kuznetsov, V. Trofimov, Geodynamics and stressed state of a subsoil of Earth (2009)

9. K. Trubetskoi, M. Lofis, S. Kuznetsov, V. Trofimov, Journal of mining science, Consultants Bureau, New York, 35-3, 209-215 (1999) 